\title{
QUANTIFICAÇÃo DE SALICILATO DE METILA EM QUATRO GÊNEROS DE Polygalaceae, POR CLAE-DAD ${ }^{\sharp}$
}

\section{José L. C. da Rocha, José F. B. Pastore}

Departamento de Biologia, Universidade Estadual de Feira de Santana, Av. Transnordestina, s/n, 44036-900 Feira de Santana BA, Brasil

\section{Hugo N. Brandão*}

Departamento de Saúde, Universidade Estadual de Feira de Santana, Av. Transnordestina, s/n, 44036-900 Feira de Santana - BA, Brasil Antonio Azeredo

Instituto de Estudos em Saúde Coletiva, Universidade Federal do Rio de Janeiro, Av. Horácio Macedo, s/n, Cidade Universitária Ilha do Fundão, 21941-598 Rio de Janeiro - RJ , Brasil

\section{Juceni P. David e Edlene O. dos Santos}

Faculdade de Farmácia, Universidade Federal da Bahia, Rua Barão de Geremoabo, s/n, Campus Univ. Ondina, 40170-290 Salvador - BA, Brasil

\section{Jorge M. David}

Instituto de Química, Universidade Federal da Bahia, Rua Barão de Geremoabo, s/n, Campus Univ. Ondina, 40170-290 Salvador - BA, Brasil

Recebido em 20/5/12; aceito em 22/9/12; publicado na web em 26/10/12

\begin{abstract}
QUANTIFICATION OF METHYL SALICYLATE IN FOUR GENERA OF THE Polygalaceae BY HPLC-DAD. Polygalaceae is represented in Brazil by ten genera and 191 species, of which the Polygala is the most representative, characterized by the occurrence of methyl salicylate. Seventeen species of Polygalaceae have been analyzed by HPLC-DAD and the technique proved to be selective, precise, accurate, and with low limits of quantification and detection. The analysis of plant material confirmed the presence of methyl salicylate, with concentration values ranging from $14.1 \mathrm{a} 126.9 \mu \mathrm{g} / \mathrm{g}$.
\end{abstract}

Keywords: HPLC; Polygalaceae; methyl salicylate.

\section{INTRODUÇÃO}

Atualmente a família Polygalaceae Hoffmanns. \& Link compreende 22 gêneros e cerca de 1.300 espécies com ampla distribuição no mundo, ${ }^{1}$ sendo as regiões temperadas quentes e tropicais seus principais centros de diversidade. ${ }^{2} \mathrm{O}$ gênero Polygala, responsável pela distribuição cosmopolita da família, compreende atualmente cerca de 725 espécies e 8 subgêneros. No Brasil, essa família está representada por 10 gêneros e 191 espécies, dos quais o gênero Polygala é o mais representativo, com 98 espécies. ${ }^{3}$ Este gênero está representado apenas pelo subgênero típico, uma vez que os outros táxons tradicionalmente reconhecidos como subgêneros estão sendo tratados como gêneros independentes. ${ }^{4}$

Espécies desta família são conhecidas por conter compostos químicos que exibem atividades analgésica, expectorante, sedativa, antifúngica, entre outras. ${ }^{5} \mathrm{~A}$ família também tem sido alvo de estudos fitoquímicos, nos quais já foram descritos saponinas, xantonas, derivados de pironas, cumarinas, ácidos graxos, fenóis e alcaloides. ${ }^{6}$ Além desses compostos, as espécies de Polygala caracterizam-se também pela presença de salicilato de metila, encontrado principalmente em suas raízes. ${ }^{7}$

O salicilato de metila é utilizado para aliviar dores em geral, principalmente, dores musculares, aplicado externamente na área afetada. Pode ser encontrado em diversas formas farmacêuticas, além de fragrâncias utilizadas em cosméticos, perfumes finos, xampus, papel higiênico, sabonetes e outros artigos de higiene pessoal, bem como em produtos não cosméticos, tais como produtos de limpeza e detergentes domésticos. ${ }^{8}$

*e-mail: hugo@uefs.br

"Artigo em homenagem ao Prof. Otto R. Gottlieb (31/8/1920-19/6/2011)
A aplicação da cromatografia a líquidos de alta eficiência (CLAE) para a análise de compostos voláteis ainda é limitada. Sendo que, nos casos de análise de compostos voláteis, a cromatografia a gás acoplada à espectrometria de massas (CG-EM) é a técnica geralmente utilizada. Porém, em estudo realizado por Villa e colaboradores, ${ }^{9}$ com salicilato de benzila e outros compostos voláteis, foi demonstrado que a CLAE também é eficiente. O estudo de Villa também demonstrou que a CLAE pode ser considerada uma técnica simples, rápida, econômica e reprodutível, mesmo para este tipo de análise. Baseado nos dados obtidos por Villa e na ausência de trabalhos com análise de salicilato de metila por CLAE, assim como de estudos quantitativos desse metabólito em espécies de Polygala, o presente trabalho apresenta a metodologia desenvolvida e validada usando CLAE-DAD para quantificação de salicilato de metila em espécies de Polygalaceae. Assim, foi observado que com os parâmetros utilizados de seletividade, linearidade, precisão, exatidão, limites de detecção e de quantificação, a metodologia aplicada atende a finalidade pretendida. Foi possível quantificar salicilato de metila nas 17 espécies analisadas, o que demonstrou grande variação na concentração de salicilato de metila nas diferentes espécies analisadas, com valores menores que $14,1 \mu \mathrm{g} / \mathrm{g}$ a maiores que $126,9 \mu \mathrm{g} / \mathrm{g}$.

\section{PARTE EXPERIMENTAL}

\section{Coleta}

A coleta foi realizada em áreas do campus da Universidade Estadual de Feira de Santana, no período da manhã, sendo que a análise do material foi realizada logo em seguida. Ressalta-se que algumas espécies foram coletadas na região de Chapada Diamantina - BA, sendo esse material conservado em saco plástico fechado e analisado 
apenas 1 dia após a coleta. As informações de coleta encontram-se na Tabela 1 e estão disponíveis no endereço eletrônico do species link, http://www.splink.org.br/index. A coleta e identificação dos espécimes foram realizadas pelo especialista nesta família botânica J. F. B. Pastore. Todos os materiais coletados para análise fitoquímica tiveram material testemunho depositado no Herbário da Universidade Estadual de Feira de Santana - HUEFS. Foram amostradas espécies de 4 gêneros diferentes, Asemeia Raf. emmend. Small, Bredemeyera Willd., Polygala L. e Securidaca L. entre os 7 gêneros de Polygalaceae representados na região da Caatinga.

\section{Materiais e equipamentos}

Os materiais empregados foram salicilato de metila Vetec ${ }^{\circledR}$, dosagem mínima 99\%; acetonitrila grau HPLC; água purificada obtida através de Milli-Q para uso em CLAE. Os demais reagentes e solventes utilizados foram de grau analítico.

Os equipamentos empregados foram sistema HPLC EZChrom Elite, consistindo de bomba VRW Hitachi L-2130, equipado com injetor e detector UV de arranjo de diodos (DAD) VRW Hitachi L-2455, e forno VRW Hitachi L-2300, coluna LiChroCART Purospher $\operatorname{Star}^{\circledR}$ RP18-e (250 mm x 4,6 mm i.d.) $(5 \mu \mathrm{m})$ (Merck, Darmastad, Germany) combinada com pré-coluna LiChroCART 4-4 LiChrospher 100RP18 (5 $\mu \mathrm{m})$ da Merck.

\section{Preparação das amostras}

As impurezas, como terra e folhas de outras plantas, foram retiradas. As raízes foram separadas das partes aéreas para a realização da análise separadamente. Alguns espécimes, devido à pequena quantidade de material vegetal conseguido, foram analisados por inteiro, sem separação das suas partes. Tanto as raízes quanto as partes aéreas foram rasuradas e pesadas. Em seguida, foram transferidas para recipientes, nos quais foi adicionado o solvente extrator (hexano), na proporção de $10 \mathrm{~mL}$ para cada $1 \mathrm{~g}$ de material vegetal. Os recipientes foram devidamente vedados e submetidos à extração por sonicação durante $1 \mathrm{~h}$, à temperatura ambiente. Após filtração, 1 $\mathrm{mL}$ do extrato foi submetido à filtração em cartucho de extração em

Tabela 1. Quantificação de salicilato de metila nas amostras

\begin{tabular}{|c|c|c|c|c|c|}
\hline Material testemunho & Data & Local de coleta & Espécies & Parte da planta & $\mu \mathrm{g}$ salicilato/g planta \\
\hline Pastore 2683 & jun. 2009 & UEFS & Asemeia ovata (Poir.) J.F.B.Pastore \& J.R.Abbott & Parte aérea & $49,33 \pm 5,41$ \\
\hline Pastore 2683 & jun. 2009 & UEFS & Asemeia ovata & Raiz & $97,72 \pm 6,30$ \\
\hline Pastore et al. 2682 & jun. 2009 & UEFS & Asemeia violacea (Aubl.) J.F.B.Pastore \& J.R.Abbott & Parte aérea & $>126,9$ \\
\hline Pastore et al. 2682 & jun. 2009 & UEFS & Asemeia violacea & Raiz & $>126,9$ \\
\hline Harley \& Pastore 56180 & set./out. 2010 & Chapada Diamantina & Bredemeyera hebeclada (DC.) J.F.B.Pastore & Caule & - \\
\hline Harley \& Pastore 56180 & set./out. 2010 & Chapada Diamantina & Bredemeyera hebeclada & Folha & - \\
\hline Pastore et al. 3205 & set./out. 2010 & Chapada Diamantina & Bredemeyera martiana A.W.Benn. & Caule & $<14,1$ \\
\hline Pastore et al. 3205 & set./out. 2010 & Chapada Diamantina & Bredemeyera martiana & Raiz & $<14,1$ \\
\hline Pastore et al. 3205 & set./out. 2010 & Chapada Diamantina & Bredemeyera martiana & Folha & - \\
\hline Harley \& Pastore & set./out. 2010 & Chapada Diamantina & Polygala aff. harleyi Marques & Planta inteira & $<14,1$ \\
\hline Pastore et al. 2687 & jun. 2009 & UEFS & Polygala boliviensis A.W.Benn. & Parte aérea & $106,12 \pm 7,92$ \\
\hline Pastore et al. 2687 & jun. 2009 & UEFS & Polygala boliviensis & Raiz & $>126,9$ \\
\hline Pastore et al. 2685 & jun. 2009 & UEFS & Polygala glochidiata Kunth & Parte aérea & - \\
\hline Pastore et al. 2685 & jun. 2009 & UEFS & Polygala glochidiata & Raiz & $>126,9$ \\
\hline Harley \& Pastore & set./out. 2010 & Chapada Diamantina & Polygala harleyi Marques var. harleyi & Planta inteira & $<14,1$ \\
\hline Harley \& Pastore 55169 & set./out. 2010 & Chapada Diamantina & Polygala harleyi var. intermedia Marques & Planta inteira & $<14,1$ \\
\hline Pastore et al. 3209 & set./out. 2010 & Chapada Diamantina & Polygala obovata A.St.-Hil. \& Moq. & Parte aérea & $<14,1$ \\
\hline Pastore et al. 3209 & set./out. 2010 & Chapada Diamantina & Polygala obovata & Raiz & $87,76 \pm 0,51$ \\
\hline Pastore et al. 2686 & jun. 2009 & UEFS & Polygala paniculata $\mathrm{L}$. & Parte aérea & $24,00 \pm 6,96$ \\
\hline Pastore et al. 2686 & jun. 2009 & UEFS & Polygala paniculata & Raiz & $>126,9$ \\
\hline Harley \& Pastore 55802 & set./out. 2010 & Chapada Diamantina & Polygala poaya Mart. & Brotos & $<14,1$ \\
\hline Harley \& Pastore 55802 & set./out. 2010 & Chapada Diamantina & Polygala poaya & Caule & $<14,1$ \\
\hline Harley \& Pastore 55802 & set./out. 2010 & Chapada Diamantina & Polygala poaya. & Flor & $<14,1$ \\
\hline Harley \& Pastore 55802 & set./out. 2010 & Chapada Diamantina & Polygala poaya & Folha & $<14,1$ \\
\hline Harley \& Pastore 55802 & set./out. 2010 & Chapada Diamantina & Polygala poaya & Raiz & $<14,1$ \\
\hline Harley \& Pastore 55802 & set./out. 2010 & Chapada Diamantina & Polygala poaya & Ramos velhos & - \\
\hline Harley \& Pastore 55814 & set./out. 2010 & Chapada Diamantina & Polygala sericea Mart. ex A.W.Benn. & Raiz & $40,61 \pm 0,22$ \\
\hline Harley \& Pastore 55814 & set./out. 2010 & Chapada Diamantina & Polygala sericea & Parte aérea & $<14,1$ \\
\hline Pastore et al. 3213 & set./out. 2010 & Chapada Diamantina & Polygala sincorensis Chodat & Parte aérea & $<14,1$ \\
\hline Pastore et al. 3213 & set./out. 2010 & Chapada Diamantina & Polygala sincorensis & Raiz & $>126.9$ \\
\hline Pastore et al. 2684 & jun. 2009 & UEFS & Polygala trichosperma Jacq. & Parte aérea & $<14,1$ \\
\hline Pastore et al. 2684 & jun. 2009 & UEFS & Polygala trichosperma & Raiz & $18,94 \pm 0,17$ \\
\hline Pastore et al. 3201 & set./out. 2010 & Chapada Diamantina & Securidaca diversifolia (L.) S.F.Blake & Caule & $<14,1$ \\
\hline Pastore et al. 3201 & set./out. 2010 & Chapada Diamantina & Securidaca diversifolia & Folha & $<14,1$ \\
\hline Pastore et al. 3201 & set./out. 2010 & Chapada Diamantina & Securidaca diversifolia & Flor & $<14,1$ \\
\hline Harley \& Pastore 55584 & set./out. 2010 & Chapada Diamantina & Securidaca tomentosa A.St.-Hil. \& Moq. & Caule & $<14,1$ \\
\hline Harley \& Pastore 55584 & set./out. 2010 & Chapada Diamantina & Securidaca tomentosa & Folha & $<14,1$ \\
\hline
\end{tabular}

* (-) - traço 
fase sólida (Solid-Phase Extraction - SPE), com $4 \mathrm{~mL}$ de acetonitrila grau HPLC. 1,5 mL da solução de acetonitrila obtida foi novamente filtrada através de membrana microporosa $(0,22 \mu \mathrm{m})$ diretamente para vial e, posteriormente, injetada no cromatógrafo.

\section{Condições da CLAE-DAD}

O volume de injeção foi de $20 \mu \mathrm{L}$ e as condições de análise incluíram gradiente de eluição com fase móvel de acetonitrila $(\mathrm{ACN})$ e água $\left(\mathrm{H}_{2} \mathrm{O}\right)$. Inicialmente, foi testado o método para separação de salicilato de benzila utilizado por Villa. Em seguida, desenvolveu-se o gradiente descrito na Tabela 2. O forno de colunas operou em $25^{\circ} \mathrm{C}$ e outras condições estão apresentadas na Tabela 2.

Tabela 2. Condições cromatográficas

\begin{tabular}{cccc}
\hline Tempo $(\mathrm{min})$ & Fluxo $(\mathrm{mL} / \mathrm{min})$ & $\mathrm{ACN}(\%)$ & $\mathrm{H}_{2} \mathrm{O}(\%)$ \\
\hline 0,0 & 0,7 & 50,0 & 50,0 \\
5,0 & 0,7 & 50,0 & 50,0 \\
15,0 & 1,0 & 60,0 & 40,0 \\
15,1 & 1,0 & 90,0 & 10,0 \\
18,0 & 1,0 & 90,0 & 10,0 \\
18,1 & 1,0 & 50,0 & 50,0 \\
22,0 & 1,0 & 50,0 & 50,0 \\
\hline
\end{tabular}

A leitura do detector de arranjo de diodo foi realizada na faixa de 210 a $400 \mathrm{~nm}$ e a aquisição cromatográfica definida em $236 \mathrm{~nm}$. A identificação foi conduzida pela comparação dos tempos de retenção e do gráfico de absorbância no UV correspondente com o sinal do padrão de referência.

\section{Método analítico da validação}

O método foi validado de acordo com os parâmetros descritos pelo International Conference on Harmonization (ICH), pela Agência Nacional de Vigilância Sanitária (ANVISA) - Resolução ANVISA RE n 899, de 29/05/2003 - e pelo Instituto Nacional de Metrologia, Normalização e Qualidade Industrial (INMETRO) - documento INMETRO DOQ-CGCRE-008, de março/2003. ${ }^{10}$ Os parâmetros utilizados foram seletividade, linearidade, precisão, exatidão, limites de detecção e de quantificação.

A seletividade foi determinada por meio da comparação de picos do padrão e das amostras, levando-se em consideração o tempo de retenção, espectro no ultravioleta e pureza do pico em 3 pontos diferentes.

A linearidade foi determinada pela curva de calibração, levando-se em consideração o coeficiente de correlação $\left(R^{2}\right)$. A curva de calibração foi obtida por injeções em triplicata de 7 soluções de diferentes concentrações do padrão externo, em uma faixa de 14,1$126,9 \mu \mathrm{g} / \mathrm{mL}$. A solução estoque do padrão externo foi preparada pela dissolução em acetonitrila do composto padrão em balão volumétrico à concentração de aproximadamente $10 \mathrm{mg} / \mathrm{mL}$.

A precisão foi determinada pela injeção em triplicata de 3 soluções padrão de salicilato de metila. Esse parâmetro foi expresso como o desvio padrão relativo.

A exatidão foi verificada pelo fator de recuperação. Amostras da matriz foram fortificadas com 3 soluções padrão de concentrações conhecidas $(125,70$ e $20 \mu \mathrm{g} / \mathrm{mL})$. As amostras fortificadas, juntamente com amostra de matriz não fortificada, foram submetidas a todo o processo de extração e injetadas em CLAE.

O limite de detecção (LD) e o limite de quantificação (LQ) foram estimados pela relação do desvio padrão e da inclinação da curva de calibração.

\section{RESULTADOS E DISCUSSÃO}

\section{Seletividade}

A especificidade do método, bem como a identificação do salicilato de metila, foi verificada pela sobreposição do cromatograma e do espectro no UV obtido para o padrão e para as amostras, comparando-se o tempo de retenção e a absorção no espectro de UV $\left(\lambda_{\max }\right)$.

A média dos tempos de retenção do padrão de salicilato de metila foi de $15,56 \pm 0,06 \min (n=5)$, enquanto que para as amostras foi observado o tempo médio de 15,12 \pm 0,26 min. A diferença entre os tempos de retenção do padrão e das amostras pode ser atribuída ao efeito de matriz inerente à amostra analisada e às diferentes concentrações dos analitos. Os dados fornecidos pelos espectros no UV corroboram a comprovação de que o composto, nos tempos de retenção em questão, se trata do salicilato de metila. Os espectros no UV apresentaram as mesmas absorções tanto para o padrão quanto para a amostra, o que corrobora a demonstração da especificidade do método.

A pureza do pico também foi estimada para comprovar a especificidade do método. Para isso, foram obtidos os espectros UV em três pontos distintos do pico $(15,47 ; 15,56$ e 15,65 min) (Tabela 3$)$.

Tabela 3. Índice de similaridade

\begin{tabular}{cccc}
\hline \multicolumn{2}{c}{ Padrão } & \multicolumn{2}{c}{ Amostra } \\
\hline $\begin{array}{c}\text { Tempo de } \\
\text { retenção }\end{array}$ & $\begin{array}{c}\text { Índice de } \\
\text { similaridade }\end{array}$ & $\begin{array}{c}\text { Tempo de } \\
\text { retenção }\end{array}$ & $\begin{array}{c}\text { Índice de } \\
\text { similaridade }\end{array}$ \\
\hline 15,56 min* $^{*}$ & 1,0000 & $14,96 \mathrm{~min}^{*}$ & 1,0000 \\
$15,47 \mathrm{~min}$ & 0,9965 & $14,87 \mathrm{~min}$ & 0,9923 \\
$15,65 \mathrm{~min}$ & 0,9987 & $15,08 \mathrm{~min}$ & 0,9932 \\
\hline * & & &
\end{tabular}

Diante dos espectros e índices de similaridade obtidos, pode-se inferir que o método consegue separar especificamente a substância de interesse.

\section{Linearidade e precisão}

$\mathrm{O}$ método proposto foi avaliado quanto à sua linearidade por meio do tratamento matemático dos resultados obtidos nas análises realizadas em triplicatas de 7 soluções padrão de concentrações conhecidas, na faixa de 14,1-126,9 $\mu \mathrm{g} / \mathrm{mL}$. A curva de calibração mostrou-se adequadamente linear, como demonstra o coeficiente de correlação de $\mathrm{R}^{2}=0,9933$.

A precisão do método foi determinada pelo desvio padrão relativo de 3 soluções padrão injetados em triplicata. Os resultados obtidos próximos a $1 \%$ demonstram a precisão do método (Tabela 4).

Tabela 4. Desvio padrão relativo

\begin{tabular}{cc}
\hline $\begin{array}{c}\text { Média das concentrações }(\mu \mathrm{g} / \mathrm{mL}) \\
\pm \text { Desvio padrão }\end{array}$ & Desvio padrão relativo \\
\hline $17,22 \pm 0,21$ & $0,45 \%$ \\
$67,88 \pm 1,42$ & $1,90 \%$ \\
$126,38 \pm 1,06$ & $0,73 \%$ \\
\hline
\end{tabular}

\section{Exatidão}

A exatidão foi avaliada por meio da porcentagem de recuperação de salicilato de metila, através da adição de soluções padrão em triplicata de concentrações 125,70 e $20 \mu \mathrm{g} / \mathrm{mL}$ adicionadas na matriz das partes aéreas. A análise dos dados da exatidão também 
contribuiu na comprovação da identidade do composto de interesse, com a coinjeção do padrão adicionado na amostra e a quantidade do próprio salicilato de metila já presente na matriz. As taxas de recuperação são demonstradas na Tabela 5 , na qual se verifica, através dos resultados obtidos, a exatidão do método utilizado.

Tabela 5. Taxa de recuperação

\begin{tabular}{ccc}
\hline $\begin{array}{c}\text { Concentração } \\
\text { adicionada }(\mu \mathrm{g} / \mathrm{mL})\end{array}$ & $\begin{array}{c}\mu \mathrm{g} \text { de salicilato/ } \\
\mathrm{g} \text { de planta }\end{array}$ & $\begin{array}{c}\text { taxa de } \\
\text { recuperação }\end{array}$ \\
\hline 0 & 119,57 & 0 \\
20 & 123,76 & $83,8 \%$ \\
70 & 133,65 & $80,5 \%$ \\
125 & 148,03 & $91,1 \%$ \\
\hline
\end{tabular}

As taxas de recuperação obtidas encontram-se dentro da faixa estabelecida pela ANVISA (80-120\%), o que comprova a exatidão do método. As diferenças dos valores obtidos devem-se, principalmente, à integração dos picos de salicilato de metila, a qual foi feita manualmente, o que aumenta o erro associado à própria manipulação.

\section{Limite de detecção e de quantificação}

O LD calculado para o método demonstrou que o mesmo se mostra bastante sensível para a detecção do componente de interesse, sendo estabelecido em 4,27 $\mu \mathrm{g} / \mathrm{mL}$. Da mesma maneira, o LQ também permitiu quantificar concentrações bastante baixas de salicilato de metila, sendo determinado em $14,24 \mu \mathrm{g} / \mathrm{mL}$.

\section{Análise das amostras}

A análise dos extratos mostrou tempo de retenção do salicilato de metila em aproxidamente 15 min (Figura 1). Os espectros no ultravioleta apresentaram-se característicos do salicilato de metila, $\operatorname{com} \lambda_{\max }$ em 237 e $303 \mathrm{~nm}$ (Figura 1).

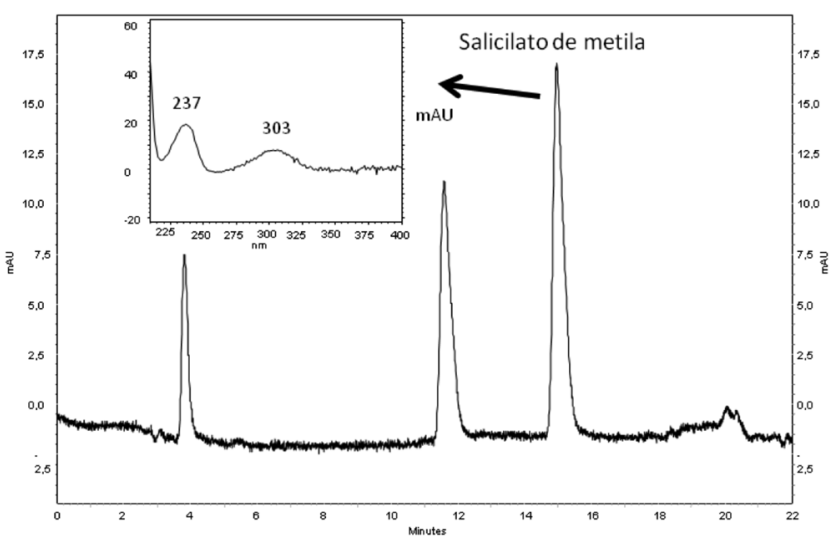

Figura 1. Perfil cromatográfico registrado em $236 \mathrm{~nm}$ de amostra das raízes de Polygala decumbens e espectro no UV do pico em 15,09

A quantificação do salicilato de metila foi determinada pela média das áreas dos picos das replicatas das amostras, como demonstrado na Tabela 1. A concentração foi determinada a partir do material fresco.

As análises mostraram grande diferença na concentração de salicilato de metila entre as espécies de Polygalaceae e nas diferentes partes das plantas. Estas diferenças podem estar relacionadas a diversos fatores, tais como o tempo de coleta e análise, hábito da planta e parte analisada da planta. A concentração do salicilato de metila no material coletado na UEFS foi maior que no material coletado na Chapada Diamantina, indicando possível perda deste metabólito durante o processo de armazenagem até a análise. Entretanto, foi observada notável exceção em Polygala sincorensis, em que a análise das raízes coletadas na região da Chapada Diamantina resultou na maior concentração de salicilato de metila dentre todas as amostras quantificadas. Outro fator relacionado pode ser o hábito das espécies, sendo que as anuais, com hábito herbáceo e raízes pouco lignificadas, resultaram em maior concentração de salicilato de metila. Nas amostras em que as raízes foram avaliadas separadamente, pode-se observar que, de modo geral, essas partes possuem maior concentração de salicilato de metila em comparação com as demais partes das plantas. Estas informações certamente estão de acordo com o que é verificado em campo, através de análises sensoriais em que o forte odor de salicilato de metila está presente nas raízes das espécies herbáceas e subarbustivas, não cespitosas, de Polygala e Asemeia.

\section{CONCLUSÃO}

O presente trabalho demonstrou que a utilização de CLAE-DAD também pode ser aplicada em compostos voláteis, sendo este o primeiro relato de quantificação de salicilato de metila por essa técnica. Anterior a esse trabalho, apenas Villa e colaboradores ${ }^{9}$ realizaram estudo semelhante envolvendo identificação de salicilato de benzila por CLAE-DAD. Além disso, no presente trabalho comprovou-se inequivocamente que essa substância é um marcador químico presente nos principais clados da família Polygalaceae.

\section{REFERÊNCIAS}

1. Marques, M. C. M.; Peixoto, A. L.; Rodriguésia 2007, 58, 95.

2. Furness, S. H.; Stafford, P. J.; Rev. Palaeobot. Palyn. 1995, 88, 61.

3. http://floradobrasil.jbrj.gov.br/2010 /FB020541, acessada em Dezembro 2011.

4. Paiva, J. A. R.; Fontqueria 1998, 50, 346; Pastore, J. F. B.; Kew Bull. 2012, 67, 2; Pastore, J. F. B.; Cardoso, D. B. S. O.; Aymard C. G. A.; Novon 2010, 20, 317

5. Lapa, F. R.; Dissertação de Mestrado, Universidade Federal do Paraná, Brasil, 2006.

6. Hamburger, M.; Gupta, M.; Hostettmann, K.; Planta Med. 1985, 51, 215; Bergeron, C.; Marston, A.; Wolfender, J. L.; Mavi, S.; Rogers, C.; Hostettmann, K.; Phytochem. Anal. 1997, 8, 32; Pinheiro, T. R.; Cechinel, V.; Santos, A. R. S.; Calixto, J. B.; Delle-Monache, F.; Pizzolatti, M. G.; Yunes, R. A.; Phytochemistry 1998, 48, 725; Pizzolatti, M. G.; Cunha Jr, A.; Pereira, W. S.; Delle Monache, F. A.; Biochem. System. Ecol. 2004, 32, 603.

7. Coelho, V. P. M.; Agra, M. F.; Baracho, G. S.; Acta Bot. Bras. 2008, 22, 225.

8. Kakker, T.; Mayersohn, M.; J. Chromatogr., B: Anal. Technol. Biomed. Life Sci. 1998, 718, 69; Davis, J. E.; J. Emerg. Med. 2007, 32, 63; Lapczynski, A.; Jones, L.; McGinty, D.; Bhatia, S. P.; Letizia, C. S.; Api, A. M.; Food Chem. Toxicol. 2007, 45, S428.

9. Villa, C.; Gambaro, R.; Mariani, E.; Dorato, S.; J. Pharm. Biomed. Anal. 2007, 44, 755 .

10. ICH; ICH Guidelines $Q 2 A$, Validation of Analytical Procedures: Definition and Terminology (CPMP/ICH/381/95), 1995; ICH Guidelines $Q 2 B$, Validation of Analytical Procedures: Methodology (CPMP/ ICH/281/95), 1995; Brasil, Ministério da Saúde, Agência Nacional de Vigilância Sanitária; Resolução n 899, de 29/05/2003, Guia para validação de métodos analíticos e bioanalíticos, Diário Oficial da União, Poder Executivo, Brasília, DF, 02/06/2003; Instituto Nacional de Metrologia, Normalização e Qualidade Industrial (Inmetro); Orientações sobre Validação de Métodos de Ensaios Químicos, DOQ-CGCRE-008, 2003 